\title{
Discussion on the Fashion Embodiment of Traditional Embroidery Technology in Modern Clothing Design
}

\author{
Wang Dandan
}

\author{
XI'AN ACADEMY OF FINE ARTS, XI'AN SHANXI CHINA \\ 479289171@qq.com
}

\begin{abstract}
:
embroidery art is one of the unique forms of expression of the chinese nation, which has important status and significance in the development of history and culture in china. In the long course of historical development, embroidery art absorbs many advanced cultural factors and has its own philosophical idea and significance. Under the background of the new period, the trend of embroidery development and promotion is changing, because embroidery is no longer a must in people's life, people's attention to embroidery is also gradually decreasing, which affects the promotion and development of traditional culture. The combination of embroidery art and clothing design can promote the inheritance and development of embroidery art, and give embroidery art a wider range of development space, but also the improvement and optimization of the traditional culture of the Chinese nation in the new period ${ }^{[1]}$.
\end{abstract}

Keywords: traditional embroidery, modern clothing design, fashion

\section{探讨传统刺绣工艺在现代服装设计中的时尚化体现}

\author{
王丹丹
}

西安美术学院 陕西省西安市 710065

479289171@qq.com

\section{摘要:}

刺绣艺术是中华民族特有的表现形式之一, 在我国的历史文化发展中国具有重要的地位和意义, 在漫 长的历史发展行程中刺绣艺术吸收了众多文化的先进因素, 并且具备了自身的哲学理念和意义。新时 期背景下, 刺绣发展和提升的趋势正在发生变化, 由于刺绣不再是人们生活中的必须品, 人们对于刺 绣的关注也在逐渐下降, 影响传统文化的弘扬和发展。将刺绣艺术与服装设计相结合, 能够推进刺绣 艺术的传承和发展，给予刺绣艺术更为宽广的发展空间，也是中华民族传统文化在新时期的完善与优 化 ${ }^{[1]}$ 。

关键词：传统刺绣；现代服装设计；时尚化

\section{1.引言}

将传统的刺绣工艺与当下的时装设计相结合, 能够 展现出时装设计的文化底蕴和艺术价值。我国在艺术创 作的过程中具有深远的历史，在长时间的文化积淀中形 成了具有中国特色的产业形式 ${ }^{[2]}$ 。

\section{2.传统刺绣概述}

\section{1 苏绣}

苏绣, 作为将江苏省苏州地区为中心囊括整个江苏 的刺绣绣作的总称。苏州位于江南地区, 自身的自然环 境和生态环境质量较好, 为苏州的丝绸生产奠定了良好 的圣体基础, 并且在太湖周围, 自然环境的优美也能够 为苏绣的形式和手法奠定良好的基础。苏州的妇女借助 良好的地理位置, 得天独厚的自然环境, 多样化的丝线, 生动形象的自然景观等等进行了优化和创作。在长期的 


\section{3 粤绣}

点, 在色彩上丰富多样、颜色明快协调; 在线条上刚劲 又不失柔美; 在针法运用上灵活多变, 富有生机, 并且 伴随着大量的绣娘在自身的工艺中精益求精, 奠定了苏 绣极强的历史文化地位。现如今在世界范围内都将苏绣 称为“东方明珠”。

在苏绣的欣赏层面上能够展现出秀品的景物远近 之分, 一动一静下似乎能够与绣品中的景物进行交流和 共通, 绣品中的人物宜喜宜嗔, 表情形象丰富, 具有生 动性, 花苗鱼虫都是生活中的常见景物, 在秀品的表现 上更是生动形象跃然纸上。

在苏绣的针法构成和使用的过程中主要是套针的 形式, 在针线相交接的位置难以明显看出针线之间的缝 隙。并且在色彩搭配上基本使用四种不一样的同类色线 亦或是相近色来进行搭配, 进而能够展现出刺绣作品的 丰富多样、色彩斑斓。在刺绣的过程中仍旧有自身独特 的发展形式, 例如在物象存在深浅较为明显的变化中, 留下一条空线。这样一来能够展现出刺绣自身的流畅感 和层次感。苏绣在花样表现的过程中形象处理较为整齐, 进而人们在对苏绣进行评价的时候通常用“平、齐、密、 细、匀、和、顺以及光”这八个字来进行表述。

\section{2 蜀绣}

蜀绣又名“川绣”，与苏绣、湘绣、粤绣齐名, 为中 国四大名绣之一。刺绣的工艺指的是在丝绸或者是其他 的布料中使用䖯丝线进行刺绣。蜀绣是我国传承时间最 长的刺绣工艺之一, 具有清丽明艳、工艺精湛的特点。

蜀绣自身的艺术底蕴较为丰富, 并且在刺绣作品中 表现出明艳动人的色彩、平整扎实的针脚、严谨认真的 技术等特点。蜀绣使用的丝线有较为粗松和较为紧致的 丝线两种, 在丝线的选择上也具有自身的特点和标准, 众多因素的共同影响下成就了蜀绣的盛名。

根据相关的统计研究表明, 蜀绣具有 130 多种, 12 类不同的刺绣形式, 在四大名绣中排名第一, 其复杂程 度和繁多工艺可见一斑。在蜀绣作品中常见的刺绣形式 有晕针、铺针、滚针、截针、掺针、沙针、盖针等。在 蜀绣中对于针脚的要求也十分详细, 需要“针脚整齐, 线 片光亮, 紧密柔和, 车拧到家” 在刺绣的过程中更是能够 使用多样化的刺绣形式, 针法变化多端, 粗细相继、虚 实相合, 能够表现出刺绣景物自身的灵动性和多样性。 这种刺绣的形式能够将花鸟鱼虫精细的雕刻出来, 在宏 观角度上能够将山水景致再现在布料上。与此同时刻画 人物的过程中也宜喜宜嗔、神态各异。经过不断的研究 和革新, 当下的蜀绣刺绣有逐渐衍生出了不同的针法, 表现动物皮毛质感的“交叉针”, 表现人物发䯽的“螺旋 针”, 表现鲤鱼鳞片的"虚实覆盖针"等, 不同的刺绣形式 能够给予蜀绣更为完善的技术, 以及更富有表现力的作 品。
粤绣是广州刺绣和潮州刺绣的总称，是中国四大名 绣之一。在唐朝阶段粤绣已经具备自身的价值和体系, 唐玄宗时, 岭南节度使张九臬进献精品刺绣给杨贵妃而 获加官三品。明代, 广东海外贸易兴盛, 明正德九年, 一葡萄牙商人在广州购得龙袍绣片回国, 并将绣袍献给 国王, 得到重赏, 粤绣从此扬名海外。

粤绣集中了潮绣的做工精湛、广绣的色彩鲜明。粤 绣展现的主要载体是戏服, 戏服的色彩表现上较为夸张 鲜明, 使用的颜色多为红色、绿色、紫色等颜色较为夺 目的色彩, 在进行刺绣布局上基本上使用较为稠密的形 式, 图案繁茂、场面热烈、用色富丽、对比强烈。布料 上基本上没有空隙, 即便是在整体构图上存在一定的空 隙, 也会使用花草、景物进行填充, 显得整体的刺绣表 现十分紧凑热闹。在粤绣发展的过程中，主要的技艺载 体是男性刺绣工人, 与其他地区的女性刺绣工人相比, 男性刺绣更喜好长针站着施绣。

粤绣在表现的过程中具有深远的历史, 在明朝中期 出现了实质性的提升, 自身的艺术表现为使用多样化的 丝线, 除丝线、线线外, 也用孔雀羽毛捻楼作线, 或用 马尾缠线作线, 刺绣作品的质感约会因为丝线种类的选 择发生自身的变化。上文中已经提到了粤绣在色彩的表 现上十分鲜艳夺目。花纹样式繁多, 并且皆为满绣。绣 品品种丰富, 有被面、枕套、床楣、披巾、头巾、绣服、 鞋帽、戏衣等，也有镜屏，挂幛、条幅等。

粤绣在刺绣的过程中针法也与以上的刺绣形式不 同, 主要的有酒插针 (即擞和针) 、套针、施毛针。使 用的布料也多为织金锻或钉金绣。刺绣作品自身的表现 力十分明显, 在众多鲜艳色彩的祄托下, 刺绣作品鲜艳 夺目、花团锦簇、轰轰烈烈, 集聚岭南风情 ${ }^{[3]}$ 。

\section{3.中国传统刺绣图案的特点}

\section{1 图案题材丰富}

在中国刺绣文化的发展具有极为深远的历史文化 背景, 即便是简单的日常图案也能够衍生出多样化的刺 绣形式。例如纹理就拥有云纹、龙纹、雷纹等不同的形 式, 纹理在刺绣作品上主要能够表现刺绣作品祥瑞如意 的理念。在进行山水花鸟图案的刺绣上能够展现自然景 物的多变和灵动。在对于人物的塑造上刺绣也具有自身 的特点, 能够将简单的人物刺绣赋予情节性和故事性, 增加了刺绣作品的精神含义。也能够一定程度上增强服 装设计进程中的创新性和时尚性 ${ }^{[4]}$ 。

\section{2 图案本身具有很强的写意特点}

古代的妇女借助良好的地理位置, 得天独厚的自然 环境, 多样化的丝线, 生动形象的自然景观等等进行了 
案已经不再是局限于刺绣中, 而是能够通过印刷等不同 的形式展现在服装设计中。并且能够在刺绣的过程中将 传统意义上的刺绣、珠绣两者进行有效融合，其中也能 够涵盖雕花、镂空等不同的表达形式，给予现代民众多 样化的审美体验。

\section{2 弱化传统图案的寓意, 注重其形式美感}

在古代社会中对于刺绣图案的引申义和内在思想 重视程度较深, 具有明显的社会等级和阶级属性, 而在 当下的社会发展进程中, 刺绣已经从多样化的社会隶属 关系中脱颖而出, 转变为符合人们审美感受的艺术形式。 通过对多样化的刺绣形式的展现, 引导人们认识到我国 传统文化的博大精深、源远流长。这种展现形式是中华 民族历史文化的二次发展和弘扬, 也是刺绣自身在新时 期中的传承和发展。

例如, 在一部分的品牌设计中大量使用了传统文化 中的龙图案, 在封建社会中这一图案的使用是具有极强 的阶级属性的, 而当下则是简单的中华文化的展现和定 位, 能够在服装设计的基础上表现出极强的民族文化, 给予我国服装设计中崭新的生机与活力。

\section{3 传统图案与现代材料的结合}

刺绣在发展的过程中具有极强的区域性和独特性, 这种特点在展现刺绣自身优势的基础上往往也限制了 刺绣的发展空间，在现阶段的服饰设计进程中，刺绣已 经不再局限于传统意义上对于布料和形式的规定中, 而 是能够在不同材质的布料上展现出来。能够为现阶段的 服装设计中增添自身的独特性和多样性。现阶段能够使 用刺绣的布料有绸缎、棉麻、皮革、特殊新型材料等等, 一定程度上拓展了刺绣自身的发展空间, 给予刺绣崭新 的发展市场。

\section{4 传统图案与现代服装款式结合}

在传统文化中不同的图案应用在不同的衣着服饰 上具有自身的特点与优势, 能够展现一定场合和时期中 对于服装的需求。但是在当下的设计过程中应当将现代 审美形式和特点与传统形式相结合, 以现代人审美观为 基础将两者进行有效融合, 从而把现代审美意识与传统 纹样背后蕴藏涵义充分展现。保证刺绣艺术在现代服饰 的设计中得到良好的应用和发展, 奠定刺绣艺术在当下 社会中的良好发展基调和空间 ${ }^{[5]}$ 。

\section{5 古今结合、中西合璧}

在中华民族几千年的发展进程中, 产生了众多具有 深刻内涵的哲学思想。其中既有代表性的是崇尚自然, 返璞归真, 追求天人合一的思想主张。在这种思想的指 
[6] Feng Yu. Application principles and forms of traditional embroidery technology in modern clothing design [J]. Art education, 2018 (12): 105-106. 和不断发展的进程中也融入了这种思想，即便是各 个历史发展阶段刺绣家崇尚的指导思想有所不同, 但是 对文字线条的和谐, 间架结构的对称, 是长久不变的理 念。刺绣中错落有致, 文墨适宜, 轻重有度的形式无一 不体现出平衡对称的美学体验 ${ }^{[6]}$ 。

克己修身的思想在儒家文化中具有重要的历史地 位, 儒家文化中讲人生划分为修身, 齐家, 治国, 平天 下几个重要的发展阶段。其中修身指的是完善自身的思 想道德水平, 是整体建设中的基础部分。刺绣发展的进 程中, 克己修身思想主要体现在以下两个方面。第一, 在刺绣学习的过程中, 想要获得更高层次上的进步应该 加强自身的练习, 排除生活中的影响和干扰, 求得刺绣 学习中的自律精神。第二, 儒家文化将人品和书品联系 在一起, 良好的思想道德水平和精神文化水平与日常的 阅读息息相关。由此可见, 刺绣学习能够有效提升人的 素养。

结束语

在刺绣与现代化服装设计相结合的过程中, 要根据 相关的历史渊源、历史进程进行合理有效的设计, 制定 相关的发展政策、发展条例, 保护法律法规, 建立健全 相关的开发和保护机制。将刺绣艺术与服装设计相结合, 能够推进刺绣艺术的传承和发展, 给予刺绣艺术更为宽 广的发展空间, 也是中华民族传统文化在新时期的完善 与优化。促进刺绣文化以合理有效的形式进行开发和利 用, 同时也应当提倡社会上的舆论分享和教育, 提升民 众对刺绣文化的重视程度, 引发民众对刺绣文化的认同 感和自豪感，有效推进刺绣文化的建设事业进程。

\section{REFERENCES}

[1] Kou Xiangqun. Application of national traditional embroidery technology in modern clothing design $[\mathrm{J}]$. Research on communication power, 2019,3 (28): 245.

[2] Yimei. On the application of Chinese traditional embroidery in modern fiber art design $[\mathrm{J}]$. Popular art, 2019 (12): 116-117.

[3] Li Ming, Liang liefeng. Inheritance and innovation of traditional embroidery technology in modern clothing design [J]. International textile guide, 2019,47 (06): 52-56.

[4] Li long, Yu Meilian. Design and application of traditional hand embroidery in modern silk clothing accessories [J]. Shandong textile economy, 2019 (04): $52-53+47$.

[5] Li Xuemei. On the inheritance and innovation of traditional embroidery crafts $[\mathrm{J}]$. Journal of Sichuan cadre correspondence college, 2018 (03): 18-20. 